Manuelle Medizin 2015 · 53:327

DOI 10.1007/s00337-015-0046-y

Online publiziert: 11 . September 2015

(c) Springer-Verlag Berlin Heidelberg 2015

CrossMark

Jürgen Meyer zu Tittingdorf

Springer Medizin, Heidelberg, Deutschland

\title{
Springer Medizin gratuliert und dankt Univ.-Prof. Dr. Hans Tilscher
}

Schon seit seiner ersten Publikation in

Die Zeitschrift Manuelle Medizin erscheint in diesem Jahr im 53. Jahrgang. Zum Zeitpunkt ihrer Gründung als „FAC Information" absolvierte Prof. Hans Tilscher gerade seine Ausbildung zum Arzt für Allgemeinmedizin, und er dürfte wenig Kenntnis von dieser Zeitschriftenneugründung in der Bundesrepublik genommen haben.

1977 kreuzten sich dann zum ersten Mal die Wege Prof. Tilschers mit der Zeitschrift Manuelle Medizin, die damals noch im Verlag für Medizin Dr. Ewald Fischer GmbH, Heidelberg, erschien. Gemeinsam mit Dr. Eder hatte Prof. Tilscher den Beitrag „Der Panoramawandel in der Betrachtungsweise von chirotherapeutischen Grundlagen " für die Zeitschrift verfasst.

Seit diesem ersten Aufsatz ist Prof. Tilscher für die Zeitschrift nicht mehr wegzudenken. Schon 4 Jahre später wurde die Zeitschrift, die bis dato „nur“ von zwei bundesdeutschen Seminaren getragen wurde, auch das offizielle Organ der Schweizer Manualmediziner sowie der Österreichischen Ärztegesellschaft für Manuelle Medizin, deren Präsident Prof. Tilscher seit 1982 ist. So war es nur konsequent, dass er in der Nachfolge von Dr. Markus Hanna schließlich auch Mitherausgeber der Zeitschrift wurde und seit 2004 in dieser Funktion die Geschicke der Zeitschrift mitverantwortet.
Manuelle Medizin hat Prof. Tilscher die Zeitschrift stets als Medium verstanden, neue Erkenntnisse, aber auch neue Ideen und Konzepte über die Grenzen Österreichs hinweg $\mathrm{zu}$ verbreiten und ihnen so den Weg in die gesamte deutschsprachige Ärzteschaft zu bahnen. Es verwundert nicht, dass seine gemeinsam mit Dr. Hanna und Dr. Graf veröffentlichte Arbeit „Klinische und röntgenologische Befunde bei der Hypermobilität und Instabilität der Lendenwirbelsäule“ aus dem Jahr $1994 \mathrm{zu}$ den wichtigsten Arbeiten der letzten 50 Jahre in Manuelle Medizin gezählt wird und daher im Jubiläumsband 50 Jahre Manuelle Medizin im Jahr 2012 noch einmal vollständig abgedruckt wurde. Neben dieser und seiner ersten Arbeit erschienen mehr als 40 Beiträge aus der Feder von Prof. Tilscher in dieser Zeitschrift, zudem betreute er zahlreiche Schwerpunktthemen und prägt durch seine Ideen und seine Fachkenntnis das Gesicht der Zeitschrift bis zum heutigen Tag. Als Verlagsredaktion können inzwischen $3 \mathrm{Ge}$ nerationen an verantwortlichen Redakteuren sich die Manuelle Medizin ohne Prof. Tilscher nicht mehr vorstellen.

Im Namen meiner Kolleginnen und Kollegen wünsche ich Ihnen, Herr Professor Tilscher, alles Gute für die Zukunft, viel Gesundheit und weiterhin den scharfen Geist, der Sie stets kennzeichnet.
Ihr erster Beitrag aus dem Jahre 1977 schließt mit der Aussage: „Manualmedizinische Untersuchung und Chirotherapie ... können für sich in Anspruch nehmen, mit dazu beigetragen zu haben, der Wirbelsäule die ihr gebührende pathogenetische Stellung einzuräumen.“ Ich denke, es trifft $\mathrm{zu}$, wenn man diesen Satz wie folgt umwandelt:

Sie, Herr Professor Tilscher, können für sich in Anspruch nehmen, mit dazu beigetragen $\mathrm{zu}$ haben, dass die manuelle Medizin ihre heutige Stellung und Akzeptanz als eine tragende Säule der Medizin innehat.

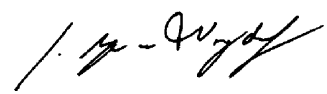

Dr. Jürgen Meyer zu Tittingdorf Head of Journals \& ePublishing 2

\section{Korrespondenzadresse}

Dr. J. Meyer zu Tittingdorf

Springer Medizin

Tiergartenstraße 17, 69121 Heidelberg,

Deutschland

juergen.meyerzutittingdorf@springer.com 